\title{
Mathematical Analysis of the Transmission Dynamics of HIV/AIDS: Role of Female Sex Workers
}

\author{
Navjot Kaur ${ }^{1, *}$, Mini Ghosh ${ }^{2}$ and S. S. Bhatia ${ }^{1}$ \\ ${ }^{1}$ School of Mathematics and Computer Applications, Thapar University, Patiala-147004, Punjab, India \\ ${ }^{2}$ School of Advanced Sciences, VIT University, Chennai Campus, Chennai 600127, Tamilnadu, India
}

Received: 18 Sep. 2013, Revised: 16 Dec. 2013, Accepted: 17 Dec. 2013

Published online: 1 Sep. 2014

\begin{abstract}
The transmission of 'Human Immunodeficiency Virus (HIV)' that causes the 'Acquired Immunodeficiency Syndrome (AIDS)' is strongly associated with un-protected sex and at the present understanding this epidemic can reach higher prevalence threshold level when there are extensive sexual contacts between the sex workers and general population. In the present work, we investigate a nonlinear model for studing the transmission dynamics of HIV/AIDS epidemic with emphasis on the role of female sex workers. Here, we consider only the heterosexual transmissions of HIV/AIDS and formulate the mathematical model by dividing the total adult population under consideration into three different classes: male, female and female sex workers. We assume different rates of recruitment for different classes of the population. The equilibria of the model and their stability are discussed in detail. The basic reproduction number $R_{0}$ of the model is computed and it is shown that the disease-free equilibrium is stable only when $R_{0}<1$. When the associated reproduction number $R_{0}>1$, the endemic equilibrium is globally stable. Finally, the numerical simulations are reported to support the presented analytical results.
\end{abstract}

Keywords: HIV, Sex-workers, Equilibrium, Stability, Numerical simulation.

\section{Introduction}

The 'Human Immunodeficiency Virus (HIV)' is a lenti-virus (i.e. a slowly replicating retrovirus) that causes 'Acquired Immunodeficiency Syndrome (AIDS)' - a condition in humans in which progressive failure of the immune system allows life-threatening opportunistic infections and cancers to infest and thrive. HIV is one of the most prevalent life threatening viruses active across the globe and more so in the developing and low income countries. Based upon documentary evidence, it was first reported in the year 1981 in an issue of the Morbidity and Morality Weekly Report (MMWR), published by the Centers for Disease Control and Prevention (CDC), USA. As per the estimates of WHO and UNAIDS [1,2] there were around 34 million people living with HIV at the end of 2011 among which 30.7 million [28.2-32.3 million] were adults and 16.7 million [15.7 - 17.6 million] were women. In the same year, around 2.5 million [2.2-2.8 million] people became newly infected, and 1.7 million died of AIDS.

As per the current scientific understanding, the infection with HIV can occur by the transfer of blood, semen, vaginal fluid, pre-ejaculate, or breast milk and within these bodily fluids HIV is present as both free virus particles and virus within infected immune cells [3]. Within the scope of this paper, we note that in the context of prostitution in developing and low income countries, the risk of female-to-male HIV transmission has been estimated as $2.4 \%$ per act and male-to-female transmission as $0.05 \%$ per act [4].

The HIV infects critically important cells (i.e. helper $\mathrm{T}$ cells - specifically cluster of differentiation (CD4+) T cells, macrophages, and dendritic cells) in the human immune system and it leads to decaying levels of the CD4+ $\mathrm{T}$ cells through a number of mechanisms including: apoptosis of uninfected bystander cells, direct viral killing of infected cells, and killing of infected CD4+ $\mathrm{T}$ cells by CD8 cytotoxic lymphocytes that recognize infected cells. And, when the CD4+ T cell numbers decline below a critical level, the cell-mediated immunity which protects against infections, is lost. The body becomes progressively more susceptible to opportunistic infections with loss of the cell-mediated immunity, e.g. illness, cancers, or neurological problems.

\footnotetext{
*Corresponding author e-mail: navjotkaur_josan@yahoo.co.in
} 
Among these infections, the 'Tubercle Bacillus (TB)' remains one of the most common life-threatening opportunistic infections which kills nearly a quarter of a million people living with HIV each year [2]. The estimates of [5] consider that there are about 2 million (range 1.0-16.2 million) female sex workers in India. In India, about $85 \%$ of new infections are associated with heterosexual transmission, especially sex workers (SWs), their clients and the sexual contacts of their clients $[6,7$, 8]. Furthermore, since most of the male clients of female SWs are married, the onward transmission of HIV is certain, specifically from HIV-positive men to their wives $[6,9]$. The overall lifetime possibility of a sex worker getting HIV infected is higher as compared to the general population which incorporates multiple risk factors, including multiple sexual partners, unprotected work conditions, barriers to negotiating the regular condom use, lack of access to appropriate health services, high prevalence of sexually transmitted infections (STIs) and sharing of injecting equipment, etc. [10]. In some of the recent studies, it has been noted that the female SWs are 13.5 times more likely to acquire HIV than all other women aged between 15-49 years, including in high HIV-prevalence countries [11,12]. The environment and context in which sex workers live and work do not support them to control the risk factors $[13,14]$. Because of these reasons, sex workers have been considered a key population and in our opinion the interventions need to be designed and implemented to guide and empower this specific population if the epidemic is to be controlled [15, 16]. The present work is motivated by this idea.

The epidemiological modelling allows us to understand the dynamics of an epidemic and to develop various control and prevention policies. It helps researchers to investigate how the system components are connected and how this connection may contribute to the growth or prevention of a specific disease. Furthermore, coupling of two or more infectious diseases can be studied via epidemiological modeling. A simple 'Susceptible-Infected-Recovered (SIR)' model of infectious diseases was developed and studied by Kermack \& McKendrick in year 1927, and that forms the foundation of almost all the epidemic models studied thereafter.

The mathematical modelling of diseases aims to improve scientific support for decision making by incorporating and analyzing the major factors responsible for the transmission of the epidemic. In the present time, mathematical models are extensively used to study epidemics to figure out the analytical conclusions based upon the epidemiological assumptions $[17,18,19,20,21$, 22,23,24,25]. Several modelling studies have been published recently that are intended to provide information to policy makers to support the development of policies and strategies for reducing the overall transmission rate of HIV infection. Numerous mathematical models have been developed to examine the role of various modes of transmission, sexual transmission of HIV incorporating effect of awareness and treatment, effect of screening of HIV infectives [17, $20,26,27,28,29]$.

In our opinion, the sex workers, their clients and regular partners are core population group which is at risk for being infected with HIV. From the available data, it has been estimated that the group sex events (GSEs) are common in developing and low income countries, e.g. Kenya, Bangladesh, India, and Argentina [30,31,32,33, 34]. The increasing testaments do suggest that the higher-risk behavior is substantially underreported in most of the HIV control and prevention surveys [35]. Therefore, in the present analysis, the attempts are made to explore the role of female sex-workers and their clients in the transmission of HIV infection.

The paper is organized: Section 2 presents the formulation of nonlinear ODE model, Section 3 describes the basic properties of the model the computation of basic reproduction number, Section 4 presents the stability analysis of the model. Section 5 reports the numerical simulations to verify our theoretical, and finally Section 6 summarizes some of the key findings of our research.

\section{Description of the model}

The total sexually active population at time $t$ denoted by $\mathrm{N}(\mathrm{t})$ or $\mathrm{N}$, is subdivide into mutually-exclusive compartments, namely HIV-susceptible male $\left(S_{m}\right)$, HIV-susceptible female $\left(S_{f}\right)$, HIV-susceptible female sex-workers $\left(S_{f s}\right)$, HIV-infected male $\left(I_{m}\right)$, HIV-infected female $\left(I_{f}\right)$, HIV-infected female sex-workers $\left(I_{f s}\right)$, AIDS-infected male $\left(A_{m}\right)$, AIDS-infected female $\left(A_{f}\right)$ and AIDS-infected female sex-workers $\left(A_{f s}\right)$. We have assumed that the AIDS patients are not participating in the transmission of viruses. $\Lambda_{1}, \Lambda_{2}, \Lambda_{3}$ denotes the recruitment rate of the population in male, female and female sex-worker classes, respectively. $\beta_{i}(i=1 ; 2 ; 3 ; 4)$ are the rates of transmission of infection from infectives to susceptibles; $\mu$ is the natural death rate constant; $d$ is the disease induced mortality rate in AIDS classes $\left(A_{m} ; A_{f} ; A_{f s}\right) ; b_{1}, b_{2}, b_{3}$ are the progression rates from HIV infective male, female and female sex-workers to respective AIDS class. Above mentioned variables, parameters and assumptions result in the following deterministic system of non-linear differential equations 
that describe the model:

$$
\begin{aligned}
& \dot{S_{m}}=\Lambda_{1}-\beta_{1} S_{m} I_{f}-\beta_{2} S_{m} I_{f s}-\mu S_{m} \\
& \dot{I_{m}}=\beta_{1} S_{m} I_{f}+\beta_{2} S_{m} I_{f s}-\left(b_{1}+\mu\right) I_{m} \\
& \dot{A_{m}}=b_{1} I_{m}-(\mu+d) A_{m} \\
& \dot{S_{f}}=\Lambda_{2}-\beta_{3} S_{f} I_{m}-\mu S_{f} \\
& \dot{I_{f}}=\beta_{3} S_{f} I_{m}-\left(b_{2}+\mu\right) I_{f} \\
& \dot{A_{f}}=b_{2} I_{f}-(\mu+d) A_{f} \\
& \dot{S_{f s}}=\Lambda_{3}-\beta_{4} S_{f s} I_{m}-\mu S_{f s} \\
& \dot{I_{f s}}=\beta_{4} S_{f s} I_{m}-\left(b_{3}+\mu\right) I_{f s} \\
& \dot{A_{f s}}=b_{3} I_{f s}-(\mu+d) A_{f s} \\
& N(t)=S_{m}+S_{f}+S_{f s}+I_{m}+I_{f}+I_{f s}+A_{m}+A_{f}+A_{f s} .
\end{aligned}
$$

Here $S_{m}>0, S_{f}>0, \quad S_{f s}>0, I_{m} \geq 0, \quad I_{f} \geq 0, \quad I_{f s} \geq$ $0, A_{m} \geq 0, \quad A_{f} \geq 0$ and $A_{f s} \geq 0$.

The flow diagram of the disease dynamics is shown in Figure 1.

\section{Analysis of the model}

\subsection{Positivity and Boundedness of the Solutions}

Theorem 3.1. For all time $t \geq 0$, all the solutions of the system (1.1) are eventually confined in the compact subset

$\Omega=\left\{\left(S_{m}, S_{f}, S_{f s}, I_{m}, I_{f}, I_{f s}, A_{m}, A_{f}, A_{f s}\right) \in \mathbb{R}_{+}^{9}: N=\right.$ $\left(S_{m}(t)+S_{f}(t)+S_{f s}(t)+I_{m}(t)+I_{f}(t)+I_{f s}(t)+A_{m}(t)+\right.$ $\left.\left.A_{f}(t)+A_{f s}(t)\right) \leq \frac{\Lambda}{\mu}\right\}$

Proof.

$\left(S_{m}(t), S_{f}(t), S_{f s}(t), I_{m}(t), I_{f}(t), I_{f s}(t), A_{m}(t), A_{f}(t), A_{f s}(t)\right)$ be any solution with positive initial conditions.

We have,

$N(t)=S_{m}(t)+S_{f}(t)+S_{f s}(t)+I_{m}(t)+I_{f}(t)+I_{f s}(t)+$ $A_{m}(t)+A_{f}(t)+A_{f s}(t)$.

The time derivative of $N(t)$ along the solution of (1.1) is $\frac{d N}{d t}=\Lambda_{1}+\Lambda_{2}+\Lambda_{3}-\mu N-d\left(A_{m}+A_{f}+A_{f s}\right)$ $\leq\left(\Lambda_{1}+\Lambda_{2}+\Lambda_{3}\right)-\mu N$, i.e. $\frac{d N}{d t}+\mu N \leq \Lambda$,

where $\Lambda=\Lambda_{1}+\Lambda_{2}+\Lambda_{3}$, the total recruitment rate in the population under consideration. Using theory of differential equations, we get $N \leq \frac{\Lambda}{\mu}\left(1-e^{-\mu t}\right)+N_{0} e^{-\mu t}$, and for $t \rightarrow \infty$, we have

$$
\lim _{t \rightarrow \infty} \sup N \leq \frac{\Lambda}{\mu}
$$

Clearly, it has been proved that all the solutions of (1.1) which initiate in $\mathbb{R}_{+}^{9}$ confined in the region $\Omega$ i.e. solutions are bounded in the interval $[0, \infty)$.

Now we will discuss the existence of all possible equilibria of the model system (1.1). We found that the system (1.1) has two possible non-negative equilibria namely the disease-free equilibrium $E_{0}$ and the endemic equilibrium $E_{1}$.

\subsection{Disease-free equilibrium $E_{0}$ and the basic reproduction number $R_{0}$}

The disease-free equilibrium (DFE) is always feasible, as at this equilibrium the infection eradicates from the population. The DFE for the model system (1.1), is given by

$$
\begin{aligned}
E_{0} & =\left(S_{m}^{0}, S_{f}^{0}, S_{f s}^{0}, I_{m}^{0}, I_{f}^{0}, I_{f s}^{0}, A_{m}^{0}, A_{f}^{0}, A_{f s}^{0}\right) \\
& =\left(\frac{\Lambda_{1}}{\mu}, \frac{\Lambda_{2}}{\mu}, \frac{\Lambda_{3}}{\mu}, 0,0,0,0,0,0\right) .
\end{aligned}
$$

The threshold quantity the basic reproduction number $\left(R_{0}\right)$ measures the average number of new HIV infections generated by a single infectious in a completely susceptible population in his/her whole infectious period. Using the standard notation in Ref. [36,37], the matrices $\mathrm{F}$ and $\mathrm{V}$, for the new infection terms and the remaining transfer terms respectively, corresponding to the system (1.1) are computed as follows:

$$
\begin{gathered}
\mathscr{F}=\left(\begin{array}{c}
\beta_{1} S_{m} I_{f}+\beta_{2} S_{m} I_{f s} \\
\beta_{3} S_{f} I_{m} \\
\beta_{4} S_{f s} I_{m} \\
0 \\
0 \\
0
\end{array}\right) \text { and } \mathscr{V}=\left(\begin{array}{c}
\left(b_{1}+\mu\right) I_{m} \\
\left(b_{2}+\mu\right) I_{f} \\
\left(b_{3}+\mu\right) I_{f s} \\
-b_{1} I_{m}+(\mu+d) A_{m} \\
-b_{2} I_{f}+(\mu+d) A_{f} \\
-b_{3} I_{f s}+(\mu+d) A_{f s}
\end{array}\right) . \\
\mathrm{F}=\mathrm{Jacobian} \text { of } \mathscr{F} \text { at DFE }=\left(\begin{array}{cccccc}
0 & \beta_{1} S_{m}^{0} & \beta_{2} S_{m}^{0} & 0 & 0 & 0 \\
\beta_{3} S_{f}^{0} & 0 & 0 & 0 & 0 & 0 \\
\beta_{4} S_{f s}^{0} & 0 & 0 & 0 & 0 & 0 \\
0 & 0 & 0 & 0 & 0 & 0 \\
0 & 0 & 0 & 0 & 0 & 0 \\
0 & 0 & 0 & 0 & 0 & 0
\end{array}\right) .
\end{gathered}
$$

and

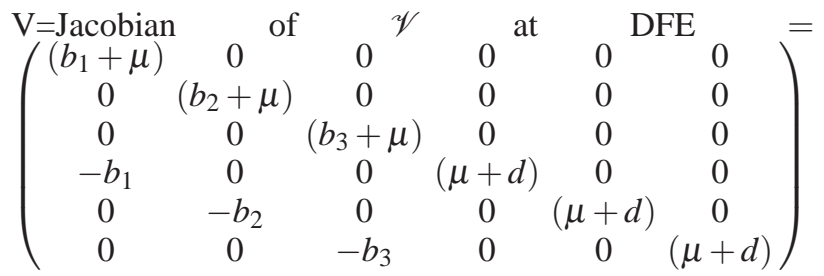

The basic reproduction number $R_{0}$ of the model system (1.1) is given by the spectral radius or the largest eigenvalue of the next generation matrix

$$
F V^{-1}=\left(\begin{array}{cccccc}
0 & \frac{\beta_{1} \Lambda_{1}}{\mu\left(b_{2}+\mu\right)} \frac{\beta_{2} \Lambda_{1}}{\mu\left(b_{3}+\mu\right)} & 0 & 0 & 0 \\
\frac{\beta_{3} \Lambda_{2}}{\mu\left(b_{1}+\mu\right)} & 0 & 0 & 0 & 0 & 0 \\
\frac{\beta_{4} \Lambda_{3}}{\mu\left(b_{1}+\mu\right)} & 0 & 0 & 0 & 0 & 0 \\
0 & 0 & 0 & 0 & 0 & 0 \\
0 & 0 & 0 & 0 & 0 & 0 \\
0 & 0 & 0 & 0 & 0 & 0
\end{array}\right)
$$

Hence $R_{0}=\sqrt{\frac{\Lambda_{1}\left\{\Lambda_{2} \beta_{1} \beta_{3}\left(b_{3}+\mu\right)+\Lambda_{3} \beta_{2} \beta_{4}\left(b_{2}+\mu\right)\right\}}{\mu^{2}\left(b_{1}+\mu\right)\left(b_{2}+\mu\right)\left(b_{3}+\mu\right)}}$. 


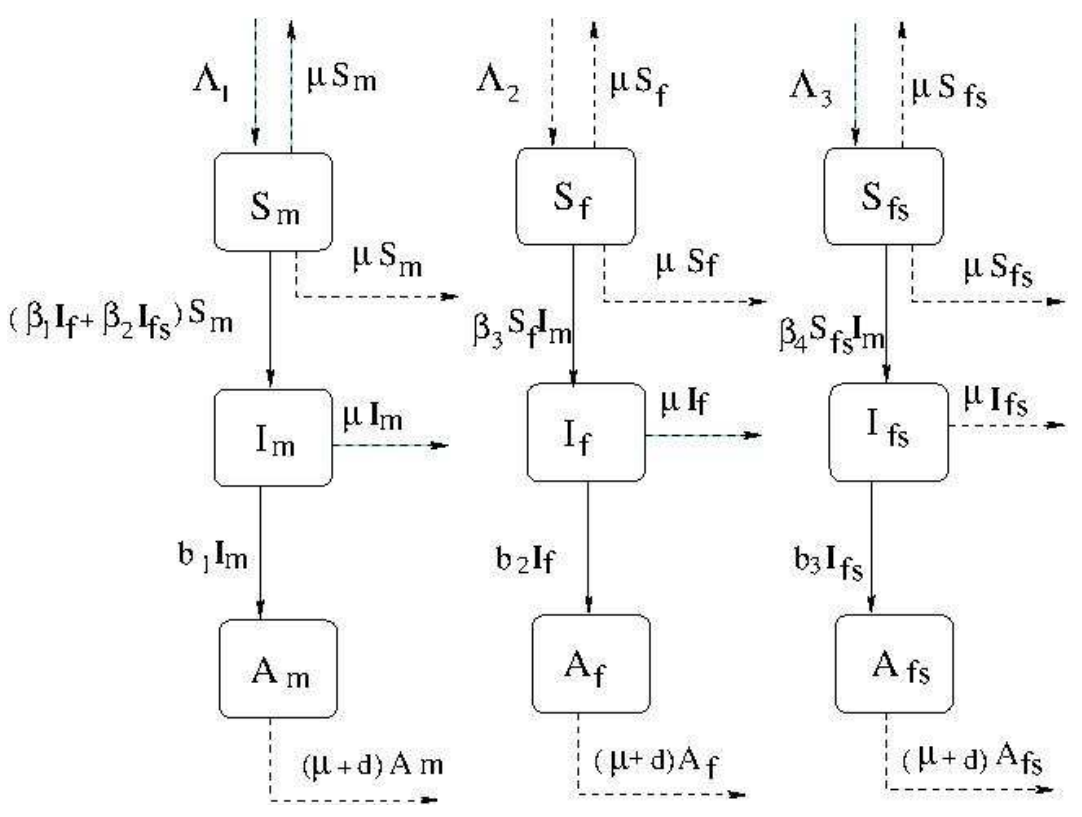

Fig. 1: Transfer Diagram of the Model (1.1)

\subsection{Existence of endemic equilibrium}

The endemic equilibrium point $E_{1}=\left(S_{m}^{*}, S_{f}^{*}, S_{f s}^{*}, I_{m}^{*}, I_{f}^{*}, I_{f s}^{*}, A_{m}^{*}, A_{f}^{*}, A_{f s}^{*}\right)$ is given as

$$
\begin{gathered}
S_{m}^{*}=\frac{\Lambda_{1}-\left(b_{1}+\mu\right) I_{m}^{*}}{\mu}, \quad S_{f}^{*}=\frac{\Lambda_{2}}{\beta I_{m}^{*}+\mu}, \quad S_{f s}^{*}=\frac{\Lambda_{3}}{\beta_{4} I_{m}^{*}+\mu}, \\
I_{f}^{*}=\frac{\Lambda_{2} \beta_{3} I_{m}^{*}}{\left(\beta_{3} I_{m}^{*}+\mu\right)\left(b_{2}+\mu\right)}, \quad I_{f s}^{*}=\frac{\Lambda_{3} \beta_{4} I_{m}^{*}}{\left(\beta_{4} I_{m}^{*}+\mu\right)\left(b_{3}+\mu\right)}, \\
A_{m}^{*}=\frac{b_{1} I_{m}^{*}}{\mu+d}, A_{f}^{*}=\frac{b_{2} \Lambda_{2} \beta_{3} I_{m}^{*}}{(\mu+d)\left(\beta_{3} I_{m}^{*}+\mu\right)\left(b_{2}+\mu\right)}, \\
A_{f s}^{*}=\frac{b_{2} \Lambda_{3} \beta_{4} I_{m}^{*}}{(\mu+d)\left(\beta_{4} I_{m}^{*}+\mu\right)\left(b_{3}+\mu\right)} .
\end{gathered}
$$

It is easy to note that each of the variables is positive if $I_{m}^{*}>0$. Here $I_{m}^{*}$ is given by the roots of the following quadratic equation,

$D_{1} I_{m}^{* 2}+D_{2} I_{m}^{*}+D_{3}=0$,

where

$$
\begin{aligned}
& D_{1}=\left[\Lambda_{2} \beta_{1}\left(b_{3}+\mu\right)+\Lambda_{3} \beta_{2}\left(b_{2}+\mu\right)\right. \\
& \left.\quad+\mu\left(b_{2}+\mu\right)\left(b_{3}+\mu\right)\right] \beta_{3} \beta_{4}\left(b_{1}+\mu\right)>0,
\end{aligned}
$$

$D_{2}=-\Lambda_{1} \Lambda_{3} \beta_{2} \beta_{3} \beta_{4}\left(b_{2}+\mu\right)-\Lambda_{1} \Lambda_{2} \beta_{1} \beta_{3} \beta_{4}\left(b_{3}+\mu\right)$

$+\Lambda_{2} \beta_{1} \beta_{3} \mu\left(b_{1}+\mu\right)\left(b_{3}+\mu\right)+\Lambda_{3} \beta_{2} \beta_{4} \mu\left(b_{1}+\mu\right)\left(b_{2}+\mu\right)$

$+\mu^{2}\left(\beta_{3}+\beta_{4}\right)\left(b_{1}+\mu\right)\left(b_{2}+\mu\right)\left(b_{3}+\mu\right)$

$=\mu^{2} \beta_{4}\left(b_{1}+\mu\right)\left(b_{2}+\mu\right)\left(b_{3}+\mu\right)$

$\times\left[1-\frac{\Lambda_{1}\left\{\Lambda_{2} \beta_{1} \beta_{3}\left(b_{3}+\mu\right)+\Lambda_{3} \beta_{2} \beta_{3}\left(b_{2}+\mu\right)\right\}}{\mu^{2}\left(b_{1}+\mu\right)\left(b_{2}+\mu\right)\left(b_{3}+\mu\right)}\right]$

$+\mu \beta_{3}\left(b_{1}+\mu\right)\left[\mu\left(b_{2}+\mu\right)\left(b_{3}+\mu\right)+\Lambda_{2} \beta_{1}\left(b_{3}+\mu\right)\right]$

$+\Lambda_{3} \mu \beta_{2} \beta_{4}\left(b_{1}+\mu\right)\left(b_{2}+\mu\right)$
$D_{3}=\mu^{3}\left(b_{1}+\mu\right)\left(b_{2}+\mu\right)\left(b_{3}+\mu\right)\left(1-R_{0}^{2}\right)<0$

Now when $R_{0}<1$ we have $D_{1}>0$, and $D_{3}>0$. Further, as the rate of transmission in female sex worker $\beta_{4}$ is always greater than or equal to the rate of transmission in susceptible female $\beta_{3}$. Hence $R_{0}<1$ implies the first bracketed term in the expression of $D_{2}$ is positive. So in this case there is no change in signs of $D_{1}, D_{2}$ and $D_{3}$. Hence Descartes's Rule of Signs ensures that there is no positive root of the above quadratic equation for $R_{0}<1$. When $R_{0}>1$, we have $D_{1}>0, D_{3}<0$ and $D_{2}$ can be positive or negative. And in this case above quadratic equation gives unique positive root irrespective of the sign of $D_{2}$. This positive root we term as $I_{m}^{*}$. Hence, existence of one positive real root $\left(I_{m}^{*}\right)$ assures the positivity of endemic equilibrium point for $R_{0}>1$.

\section{Stability Analysis}

Theorem 4.1 The disease-free equilibrium $E_{0}$, is locally asymptotically stable when $R_{0}<1$ and unstable otherwise.

Proof. To study the stability of DFE $E_{0}$ the Jacobian matrix $M_{1}$ of the system (1.1) has been calculated and it has been seen that six eigenvalues of the matrix are $-\mu,-\mu,-\mu,-(\mu+d),-(\mu+d),-(\mu+d)$ and rest of the three eigenvalues are the roots of the following cubic equation,

$$
a_{0} \lambda^{3}+a_{1} \lambda^{2}+a_{2} \lambda+a_{3}=0,
$$


where

$a_{0}=1$,

$a_{1}=x+y+z$

$a_{2}=x y+y z+z x-\frac{\beta_{1} \beta_{3} \Lambda_{1} \Lambda_{2}}{\mu^{2}}-\frac{\beta_{2} \beta_{4} \Lambda_{1} \Lambda_{3}}{\mu^{2}}$,

$a_{3}=x y z-\frac{\beta_{1} \beta_{3} \Lambda_{1} \Lambda_{2} z}{\mu^{2}}-\frac{\beta_{2} \beta_{4} \Lambda_{1} \Lambda_{3} y}{\mu^{2}}=x y z\left(1-R_{0}^{2}\right)$,

and $x=b_{1}+\mu, y=b_{2}+\mu, z=b_{3}+\mu$. Clearly $a_{1}>0, a_{3}>$ 0 for $R_{0}<1$ and $a_{1} a_{2}-a_{3}$ is given as follows:

$$
\begin{aligned}
& a_{1} a_{2}-a_{3} \\
= & (x+y+z)\left[x y+y z+z x-\frac{\beta_{1} \beta_{3} \Lambda_{1} \Lambda_{2}}{\mu^{2}}-\frac{\beta_{2} \beta_{4} \Lambda_{1} \Lambda_{3}}{\mu^{2}}\right] \\
& -x y z+\frac{\beta_{1} \beta_{3} \Lambda_{1} \Lambda_{2} z}{\mu^{2}}+\frac{\beta_{2} \beta_{4} \Lambda_{1} \Lambda_{3} y}{\mu^{2}}, \\
= & (x+y)\left[x(y+z)-\frac{\beta_{1} \beta_{3} \Lambda_{1} \Lambda_{2}}{\mu^{2}}-\frac{\beta_{2} \beta_{4} \Lambda_{1} \Lambda_{3}}{\mu^{2}}\right] \\
& +\frac{y \Lambda_{1} \Lambda_{3} \beta_{2} \beta_{4}}{\mu^{2}}+y z(y+z)+z\left[x(y+z)-\frac{\beta_{2} \beta_{4} \Lambda_{1} \Lambda_{3}}{\mu^{2}}\right] .
\end{aligned}
$$

It is easy to visualize that $a_{1} a_{2}-a_{3}$ is positive for $R_{0}<1$ as $R_{0}<1$ corresponds to $x(y+z)>\frac{\beta_{1} \beta_{3} \Lambda_{1} \Lambda_{2}}{\mu^{2}}+\frac{\beta_{2} \beta_{4} \Lambda_{1} \Lambda_{3}}{\mu^{2}}$. Hence using Routh-Hurwitz criteria, the roots of the above cubic have negative real parts. Thus DEF $E_{0}$ is locally asymptotically stable for $R_{0}<1$.

\section{Global stability of disease-free equilibrium.}

We will use the theorem by Castillo-Chavez et al. [38], to prove the global stability result.

Theorem 4.2 If the model system (1.1) can be written in the form :

$$
\left\{\begin{array}{l}
X^{\prime}(t)=F(X, Y), \\
Y^{\prime}(t)=G(X, Y), G(X, 0)=0 .
\end{array}\right.
$$

where $X=S$ and $S=\left(S_{m}, S_{f}, S_{f s}\right)^{T}$ and $Y=\left(I_{m}, I_{f}, I_{f s}, A_{m}, A_{f}, A_{f s}\right)^{T}$ with $X \in \mathbb{R}^{3}+$ denoting the number of uninfected individuals and $Y \in \mathbb{R}^{6}+$ denoting number of HIV infectives and AIDS patients.

The disease-free equilibrium is denoted here by $E_{0}=$ $\left(X_{0}, 0\right)=\left(\frac{\Lambda_{1}}{\mu}, \frac{\Lambda_{2}}{\mu}, \frac{\Lambda_{3}}{\mu}, 0\right)$

The conditions $\left(H_{1}\right)$ and $\left(H_{2}\right)$ below must be met to guarantee global asymptotic stability.

$H_{1}$ : For $X^{\prime}(t)=F\left(X_{0}, 0\right), X_{0}$ is globally asymptotically stable (g.a.s.),

$H_{2}: G(X, Y)=A Y-\widehat{G}(X, Y), \widehat{G}(X, Y) \geq 0$ for $(X, Y) \in \Omega$ here $A=D_{Y} G\left(X_{0}, 0\right)$ is an M-matrix (the off diagonal elements of A are nonnegative) and $\Omega$ is the region where the model forms biological sense. If system (1.1) satisfies the conditions mentioned in Eq. (4.1), then the fixed point $E_{0}=\left(X_{0}, 0\right)$ is a globally asymptotic stable equilibrium of model system (1.1) provided $R_{0}<1$. For system (1.1) the result is stated and proved in Theorem 4.3. given below:
Theorem 4.3 The fixed point $E_{0}=\left(X_{0}, 0\right)$ is a globally asymptotically stable equilibrium of system (1.1) provided that $R_{0}<1$ and the assumptions in Eq. (4.1) are satisfied. Proof. In Theorem 4.1 we have proved that for $R_{0}<1, E_{0}$ is locally asymptotically stable. Applying Theorem 4.2 to model system (1.1), consider

$F\left(X_{0}, 0\right)=\Gamma-\mu S$, where $\Gamma=\left(\Lambda_{1}, \Lambda_{2}, \Lambda_{3}\right)^{T}, G(X, Y)=$ $A Y-\widehat{G}(X, Y)$,

where,

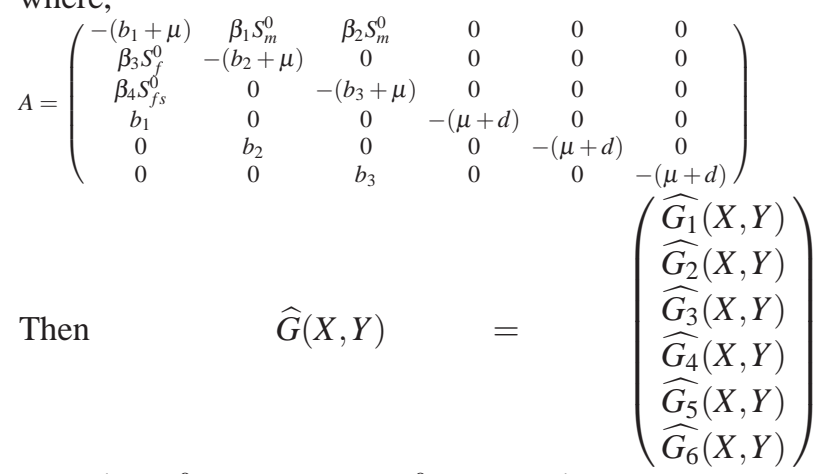

$$
=\left(\begin{array}{c}
\beta_{1}\left(S_{m}^{0}-S_{m}\right) I_{f}+\beta_{2}\left(S_{m}^{0}-S_{m}\right) I_{f s} \\
\beta_{3}\left(S_{f}^{0}-S_{f}\right) I_{m} \\
\beta_{4}\left(S_{f s}^{0}-S_{f s}\right) I_{m} \\
0 \\
0 \\
0
\end{array}\right)
$$

Here, $S_{m}^{0} \geq S_{m} ; S_{f}^{0} \geq S_{f} ; S_{f s}^{0} \geq S_{f s}$, hence it is clear that $\widehat{G}(X, Y) \geq 0$ for all $(X, Y) \in \mathbb{R}_{+}^{6}$. We also notice that the matrix $A$ is an M-Matrix since all its off-diagonal elements are non-negative. Hence, this proves the global stability of the DFE $\left(E_{0}\right)$.

Theorem 4.4 The endemic equilibrium, $E_{1}=\left(S_{m}^{*}, S_{f}^{*}, S_{f_{s}}^{*}, I_{m}^{*}, I_{f}^{*}, I_{f s}^{*}, A_{m}^{*}, A_{f}^{*}, A_{f_{s}}^{*}\right)$, of model (1.1) is globally asymptotically stable.

Proof. To prove global stability result we propose the following Lyapunov function

$$
\begin{aligned}
& V_{1}=c_{1}\left(S_{m}-S_{m}^{*}-S_{m}^{*} \ln \frac{S_{m}}{S_{m}^{*}}\right)+c_{2}\left(S_{f}-S_{f}^{*}-S_{f}^{*} \ln \frac{S_{f}}{S_{f}^{*}}\right) \\
& +c_{3}\left(S_{f s}-S_{f s}^{*}-S_{f s}^{*} \ln \frac{S_{f s}}{S_{f s}^{*}}\right)+c_{4}\left(I_{m}-I_{m}^{*}-I_{m}^{*} \ln \frac{I_{m}}{I_{m}^{*}}\right) \\
& +c_{5}\left(I_{f}-I_{f}^{*}-I_{f}^{*} \ln \frac{I_{f}}{I_{f}^{*}}\right)+c_{6}\left(I_{f s}-I_{f s}^{*}-I_{f s}^{*} \ln \frac{I_{f s}}{I_{f s}^{*}}\right) .
\end{aligned}
$$

The time derivative of $V_{1}$ is given by

$$
\begin{aligned}
& \dot{V}_{1}=c_{1}\left(1-\frac{S_{m}^{*}}{S_{m}}\right) \dot{S_{m}}+c_{2}\left(1-\frac{S_{f}^{*}}{S_{f}}\right) \dot{S_{f}}+c_{3}\left(1-\frac{S_{f s}^{*}}{S_{f s}}\right) \dot{S_{f s}} \\
& +c_{4}\left(1-\frac{I_{m}^{*}}{I_{m}}\right) \dot{I_{m}}+c_{5}\left(1-\frac{I_{f}^{*}}{I_{f}}\right) \dot{I}_{f}+c_{6}\left(1-\frac{I_{m}^{*}}{I_{f s}}\right) \dot{I_{f s}} \\
& =c_{1}\left(1-\frac{S_{m}^{*}}{S_{m}}\right)\left[\Lambda_{1}-\beta_{1} S_{m} I_{f}-\beta_{2} S_{m} I_{f s}-\mu S_{m}\right] \\
& \quad+c_{2}\left(1-\frac{S_{f}^{*}}{S_{f}}\right)\left[\Lambda_{2}-\beta_{3} S_{f} I_{m}-\mu S_{f}\right] \\
& \quad+c_{3}\left(1-\frac{S_{f s}^{*}}{S_{f s}}\right)\left[\Lambda_{3}-\beta_{4} S_{f s} I_{m}-\mu S_{f s}\right] \\
& \quad+c_{4}\left(1-\frac{I_{m}^{*}}{I_{m}}\right)\left[\beta_{1} S_{m} I_{f}+\beta_{2} S_{m} I_{f s}-\left(b_{1}+\mu\right) I_{m}\right] \\
& \quad+c_{5}\left(1-\frac{I_{f}^{*}}{I_{f}}\right)\left[\beta_{3} S_{f} I_{m}-\left(b_{2}+\mu\right) I_{f}\right]
\end{aligned}
$$




$$
+c_{6}\left(1-\frac{I_{m}^{*}}{I_{f s}}\right)\left[\beta_{4} S_{f s} I_{m}-\left(b_{3}+\mu\right) I_{f s}\right] .
$$

The model system (1.1) satisfy the following relations on the equilibrium point:

$$
\begin{aligned}
& \Lambda_{1}=\beta_{1} S_{m}^{*} I_{f}^{*}+\beta_{2} S_{m}^{*} I_{f s}^{*}+\mu S_{m}^{*}, \\
& \left(b_{1}+\mu\right)=\beta_{1} S_{m}^{*} \frac{I_{f}^{*}}{I_{m}^{*}}+\beta_{2} S_{m}^{*} \frac{I_{f s}^{*}}{I_{m}^{*}}, \\
& \Lambda_{2}=\beta_{3} S_{f}^{*} I_{m}^{*}+\mu S_{f}^{*}, \\
& \left(b_{2}+\mu\right)=\beta_{3} S_{f}^{*} \frac{I_{m}^{*}}{I_{f}^{*}}, \\
& \Lambda_{3}=\beta_{4} S_{f s}^{*} I_{m}^{*}+\mu S_{f s}^{*}, \\
& \left(b_{3}+\mu\right)=\beta_{4} S_{f s}^{*} \frac{I_{m}^{*}}{I_{f s}^{*}},
\end{aligned}
$$

Substitute the parameter values from $\Lambda_{1}$ to $\left(b_{3}+\mu\right)$ in (4.2), we obtain

$$
\begin{aligned}
& \dot{V}_{1}=c_{1}\left(1-\frac{S_{m}^{*}}{S_{m}}\right) \\
& \times\left[\beta_{1} S_{m}^{*} I_{f}^{*}+\beta_{2} S_{m}^{*} I_{f s}^{*}+\mu S_{m}^{*}-\beta_{1} S_{m} I_{f}-\beta_{2} S_{m} I_{f s}-\mu S_{m}\right] \\
& +c_{2}\left(1-\frac{S_{f}^{*}}{S_{f}}\right)\left[\beta_{3} S_{f}^{*} I_{m}^{*}+\mu S_{f}^{*}-\beta_{3} S_{f} I_{m}-\mu S_{f}\right] \\
& +c_{3}\left(1-\frac{S_{f s}^{*}}{S_{f s}}\right)\left[\beta_{4} S_{f s}^{*} I_{m}^{*}+\mu S_{f s}^{*}-\beta_{4} S_{f s} I_{m}-\mu S_{f s}\right] \\
& +c_{4}\left(1-\frac{I_{m}^{*}}{I_{m}}\right) \\
& \times\left[\beta_{1} S_{m} I_{f}+\beta_{2} S_{m} I_{f s}-\left(\beta_{1} S_{m}^{*} \frac{I_{f}^{*}}{I_{m}^{*}}+\beta_{2} S_{m}^{*} \frac{I_{f s}^{*}}{I_{m}^{*}}\right) I_{m}\right] \\
& +c_{5}\left(1-\frac{I_{f}^{*}}{I_{f}}\right)\left[\beta_{3} S_{f} I_{m}-\left(\beta_{3} S_{f}^{*} \frac{I_{m}^{*}}{I_{f}^{*}}\right) I_{f}\right] \\
& +c_{6}\left(1-\frac{I_{m}^{*}}{I_{f s}}\right)\left[\beta_{4} S_{f s} I_{m}-\left(\beta_{4} S_{f s}^{*} \frac{I_{m}^{*}}{I_{f s}^{*}}\right) I_{f s}\right] \\
& =-c_{1} \mu \frac{\left(S_{m}-S_{m}^{*}\right)^{2}}{S_{m}}-c_{2} \mu \frac{\left(S_{f}-S_{f}^{*}\right)^{2}}{S_{f}}-c_{3} \mu \frac{\left(S_{f s}-S_{f s}^{*}\right)^{2}}{S_{f s}} \\
& +c_{1}\left(1-\frac{S_{m}^{*}}{S_{m}}\right)\left(\beta_{1} S_{m}^{*} I_{f}^{*}+\beta_{2} S_{m}^{*} I_{f s}^{*}-\beta_{1} S_{m} I_{f}-\beta_{2} S_{m} I_{f s}\right) \\
& +c_{2}\left(1-\frac{S_{f}^{*}}{S_{f}}\right)\left(\beta_{3} S_{f}^{*} I_{m}^{*}-\beta_{3} S_{f} I_{m}\right) \\
& +c_{3}\left(1-\frac{S_{f s}^{*}}{S_{f s}}\right)\left(\beta_{4} S_{f s}^{*} I_{m}^{*}-\beta_{4} S_{f s} I_{m}\right) \\
& +c_{4}\left(1-\frac{I_{m}^{*}}{I_{m}}\right) \\
& \times\left[\beta_{1} S_{m} I_{f}+\beta_{2} S_{m} I_{f s}-\left(\beta_{1} S_{m}^{*} \frac{I_{f}^{*}}{I_{m}^{*}}+\beta_{2} S_{m}^{*} \frac{I_{f s}^{*}}{I_{m}^{*}}\right) I_{m}\right] \\
& +c_{5}\left(1-\frac{I_{f}^{*}}{I_{f}}\right)\left(\beta_{3} S_{f} I_{m}-\left(\beta_{3} S_{f}^{*} \frac{I_{m}^{*}}{I_{f}^{*}}\right) I_{f}\right) \\
& +c_{6}\left(1-\frac{I_{m}^{*}}{I_{f s}}\right)\left(\beta_{4} S_{f s} I_{m}-\left(\beta_{4} S_{f s}^{*} \frac{I_{m}^{*}}{I_{f s}^{*}}\right) I_{f s}\right) \\
& =-c_{1} \mu\left(S_{m}-S_{m}^{*}\right)^{2} / S_{m}-c_{2} \mu\left(S_{f}-S_{f}^{*}\right)^{2} / S_{f}-c_{3} \mu\left(S_{f s}-S_{f s}^{*}\right)^{2} \\
& / S_{f} s+f\left(x_{1}, x_{2}, x_{3}, x_{4}, x_{5}, x_{6}\right)
\end{aligned}
$$

where

$\frac{S_{m}}{S_{m}^{*}}=x_{1}, \frac{S_{f}}{S_{f}^{*}}=x_{2}, \frac{S_{f s}}{S_{f s}^{*}}=x_{3}, \frac{I_{m}}{I_{m}^{*}}=x_{4}, \frac{I_{f}}{I_{f}^{*}}=x_{5}$,

$\frac{I_{f s}}{I_{f s}^{*}}=x_{6}, S_{m}^{*} I_{f}^{*}=a, S_{f}^{*} I_{m}^{*}=b, S_{f s}^{*} I_{m}^{*}=c, S_{m}^{*} I_{f s}^{*}=d$.

We get,

$f\left(x_{1}, x_{2}, x_{3}, x_{4}, x_{5}, x_{6}\right)=\left(-c_{1} \beta_{1} a+c_{4} \beta_{1} a\right) x_{1} x_{5}+$ $\left(-c_{5} \beta_{3} b+c_{1} \beta_{1} a\right) x_{5}+\left(-c_{1} \beta_{2} d+c_{4} \beta_{2} d\right) x_{1} x_{6}+\left(c_{1} \beta_{2} d-\right.$ $\left.c_{6} \beta_{4} c\right) x_{6}+\left(-c_{2} \beta_{3} b+c_{5} \beta_{3} b\right) x_{2} x_{4}+\left(c_{2} \beta_{3} b+c_{3} \beta_{4} c-\right.$ $\left.c_{4} \beta_{1} a-c_{4} \beta_{2} d\right) x_{4}+\left(-c_{3} \beta_{4} c+c_{6} \beta_{4} c\right) x_{3} x_{4}+c_{1} \beta_{1} a-$ $c_{1} \beta_{1} a \frac{1}{x_{1}}+c_{1} \beta_{2} d-c_{1} \beta_{2} d \frac{1}{x_{1}}+c_{2} \beta_{3} b-c_{2} \beta_{3} b \frac{1}{x_{2}}+c_{3} \beta_{4} c-$ $c_{3} \beta_{4} c \frac{1}{x_{3}}-c_{4} \beta_{1} a \frac{x_{1} x_{5}}{x_{4}}-c_{4} \beta_{2} d \frac{x_{1} x_{6}}{x_{4}}+c_{4} \beta_{1} a+c_{4} \beta_{2} d-$ $c_{5} \beta_{3} b \frac{x_{2} x_{4}}{x_{5}}+c_{5} \beta_{3} b-c_{6} \beta_{4} c \frac{x_{3} x_{4}}{x_{6}}+c_{6} \beta_{4} c$.

In order to determine $c_{1}, c_{2}, c_{3}, c_{4}, c_{5}$ and $c_{6}$, we set the coefficients of $x_{1} x_{5}, x_{5}, x_{1} x_{6}, x_{6}, x_{2} x_{4}, x_{4}$ and $x_{3} x_{4}$ equal to zero and solving the resulting equations, we obtain

$$
c_{1}=c_{4} ; c_{2}=\frac{c_{4} \beta_{1} a}{\beta_{3} b}=c_{5} ; c_{3}=\frac{c_{4} \beta_{2} d}{\beta_{4} c}=c_{6} .
$$

Further by choosing $c_{1}=c_{4}=1$, we get

$$
\begin{aligned}
f\left(x_{1}, x_{2}, x_{3}, x_{4}, x_{5}, x_{6}\right)= & \beta_{1} a\left[4-\frac{1}{x_{1}}-\frac{x_{1} x_{5}}{x_{4}}-\frac{x_{2} x_{4}}{x_{5}}-\frac{1}{x_{2}}\right] \\
& +\beta_{2} d\left[4-\frac{1}{x_{1}}-\frac{x_{1} x_{6}}{x_{4}}-\frac{x_{3} x_{4}}{x_{6}}-\frac{1}{x_{3}}\right] .
\end{aligned}
$$

Since, the arithmetic mean is greater than or equal to the geometric mean, we have

$\frac{1}{x_{1}}+\frac{x_{1} x_{5}}{x_{4}}+\frac{x_{2} x_{4}}{x_{5}}+\frac{1}{x_{2}} \geq 4$ and $\frac{1}{x_{1}}+\frac{x_{1} x_{6}}{x_{4}}+\frac{x_{3} x_{4}}{x_{6}}+\frac{1}{x_{3}} \geq 4$.

Finally we obtained,

$$
\begin{aligned}
& \dot{V}_{1}=-\mu \frac{\left(S_{m}-S_{m}^{*}\right)^{2}}{S_{m}}-\mu \frac{\beta_{1} a}{\beta_{3} b} \frac{\left(S_{f}-S_{f}^{*}\right)^{2}}{S_{f}}- \\
& \mu \frac{\beta_{2} d}{\beta_{4} c} \frac{\left(S_{f s}-S_{f s}^{*}\right)^{2}}{S_{f s}}+\beta_{1} a\left[4-\frac{1}{x_{1}}-\frac{x_{1} x_{5}}{x_{4}}-\frac{x_{2} x_{4}}{x_{5}}-\frac{1}{x_{2}}\right]+ \\
& \beta_{2} d\left[4-\frac{1}{x_{1}}-\frac{x_{1} x_{6}}{x_{4}}-\frac{x_{3} x_{4}}{x_{6}}-\frac{1}{x_{3}}\right] .
\end{aligned}
$$

Thus, it implies $\dot{V}_{1} \leq 0$ in $\Omega$. The equality $\dot{V}_{1}=0$ holds only for $x_{1}=x_{2}=x_{3}=x_{4}=x_{5}=x_{6}=1$ for which $S_{m}=S_{m}^{*}, S_{f}=S_{f}^{*}, S_{f s}=S_{f s}^{*}, I_{m}=I_{m}^{*}, I_{f}=I_{f}^{*}, I_{f s}=I_{f s}^{*}$. From the LaSalles invariance principle [39], the unique endemic equilibrium $E_{1}$ of system (1.1) is globally asymptotically stable for $R_{0}>1$.

\section{Numerical simulation}

The objective of these simulations is to illustrate some of the theoretical results obtained in this paper. The system (1.1) is simulated for various set of parameters using XPPAUT [40]. In Figure 2, the stability of disease-free equilibrium point $E_{0}$ is shown for parameters values $\Lambda_{1}=80, \Lambda_{2}=60, \Lambda_{3}=50, \beta_{1}=0.00005, \beta_{2}=$ $0.0002, \beta_{3}=0.0001, \beta_{4}=0.0003, b_{1}=0.107261, b_{2}=$ $0.0924, b_{3}=0.25, \mu=0.0743, d=0.123$. The basic reproduction number for this set of parameters is 0.939145 and the disease-free equilibrium point $E_{0}$ is 


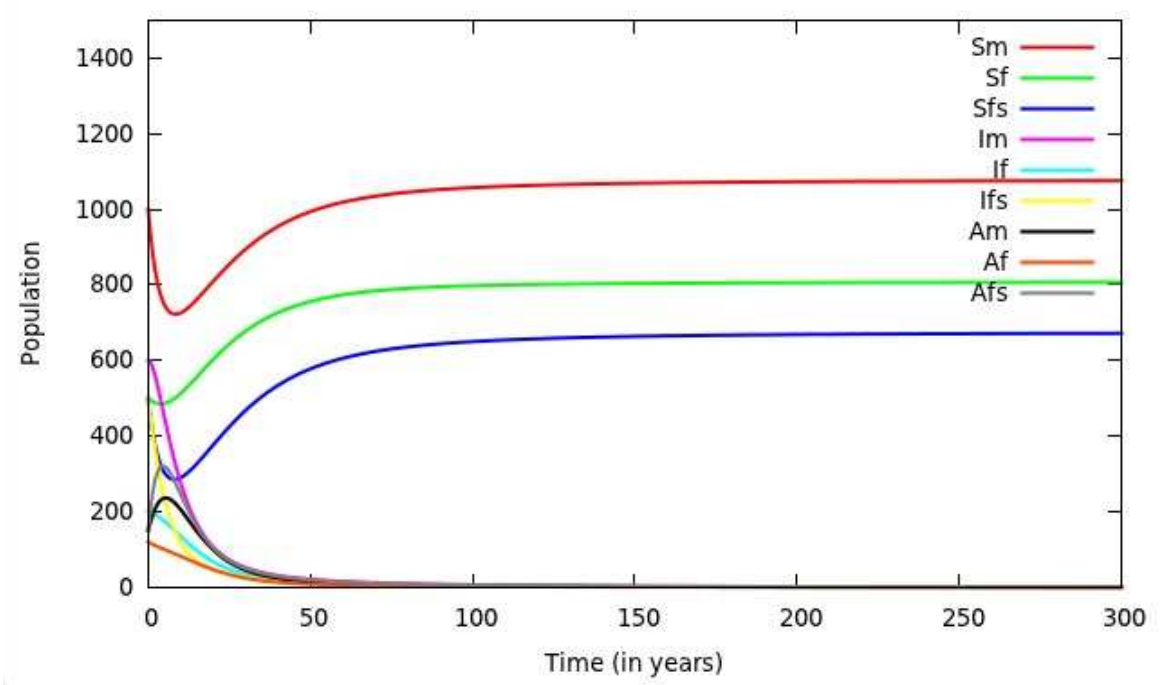

Fig. 2: Stability of the disease-free equilibrium point $E_{0}$ for $R_{0}=0.939145$.

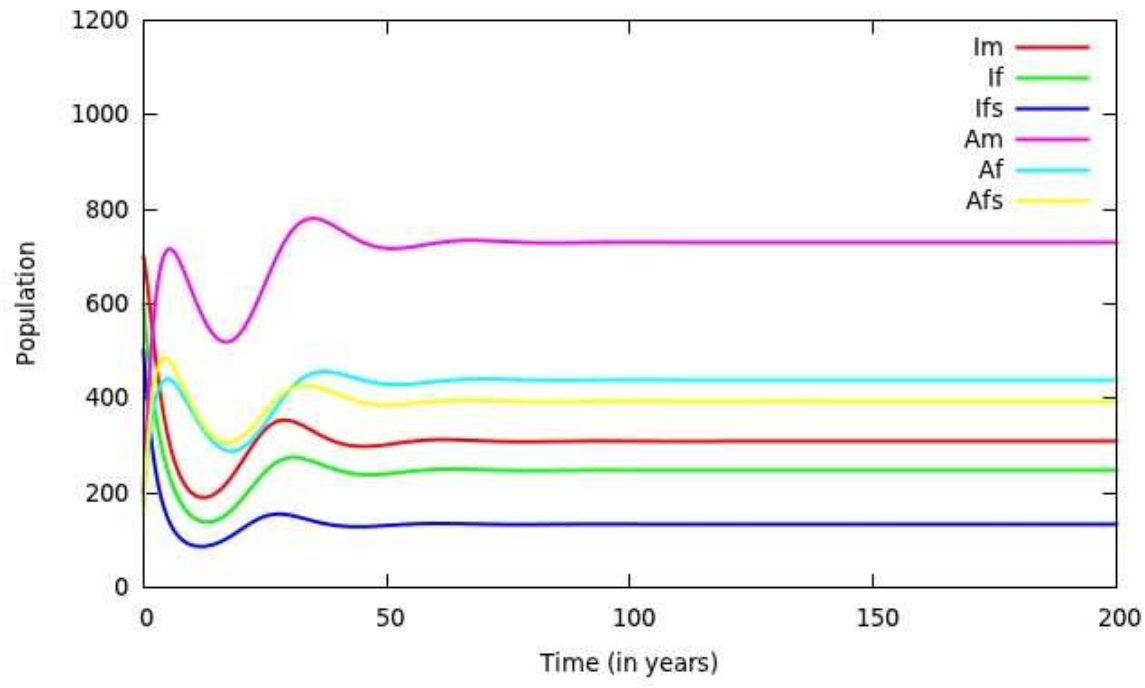

Fig. 3: Stability of the endemic equilibrium point $E_{1}$ for $R_{0}=1.460919$.

(1076.7, 807.54, 672.95, 0, 0, 0, 0, 0, 0). Here, Figure.3 corresponds to the stability of the endemic equilibrium point $E_{1}$ for the parameter values

$\Lambda_{1}=200, \Lambda_{2}=150, \Lambda_{3}=100, \beta_{1}=0.0002, \beta_{2}=$ $0.0004, \beta_{3}=0.0002, \beta_{4}=0.0004, b_{1}=0.4, b_{2}=$ $0.3, b_{3}=0.5, \mu=0.0463, d=0.123$

For this set of parameter values the basic reproduction number $R_{0}$ is evaluated as $R_{0}=1.460919$ and endemic equilibrium point is $(1341.5,1387.7,588.64,308.96$, 247.62, 133.16, 729.97, 438.77, 393.27). The phase portrait of this equilibrium point in $S_{m}-I_{m} ; S_{f}-I_{f}$ and $S_{f s}-I_{f s}$ planes are shown in Figures. $4-6$. In Figure 7, the sensitivity of different population groups (i.e. susceptible males, HIV infective males, AIDS infected males, AIDS infected female, AIDS infected female sex workers) is shown for different values of $\beta_{2}$. It is observed that the number of HIV infectives increases with the increase in this parameter $\beta_{2}$, which corresponds to the rate of transmission due to female sex-workers.

\section{Discussion}

In developing and low income countries, it has been widely reported that the female sex workers are one of the most vulnerable groups in transmission of the HIV. As the HIV transmission is strongly associated with un-protected sex, the WHO has framed guidelines [10] for prevention 


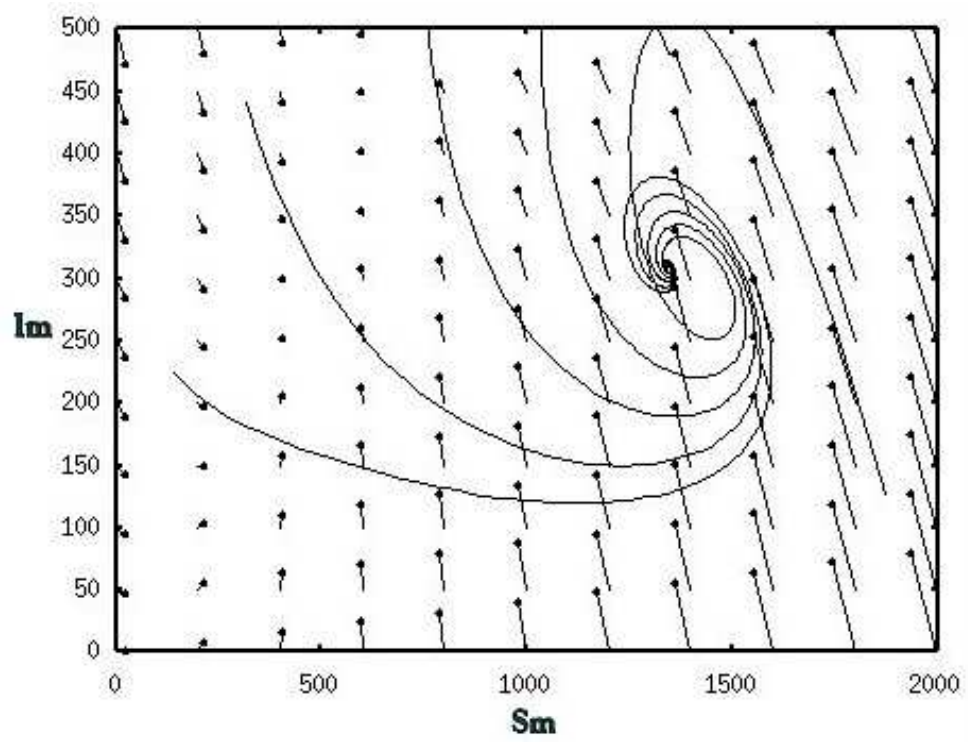

Fig. 4: The phase portrait of endemic equilibrium point $E_{1}$ in $S_{m}-I_{m}$ plane.

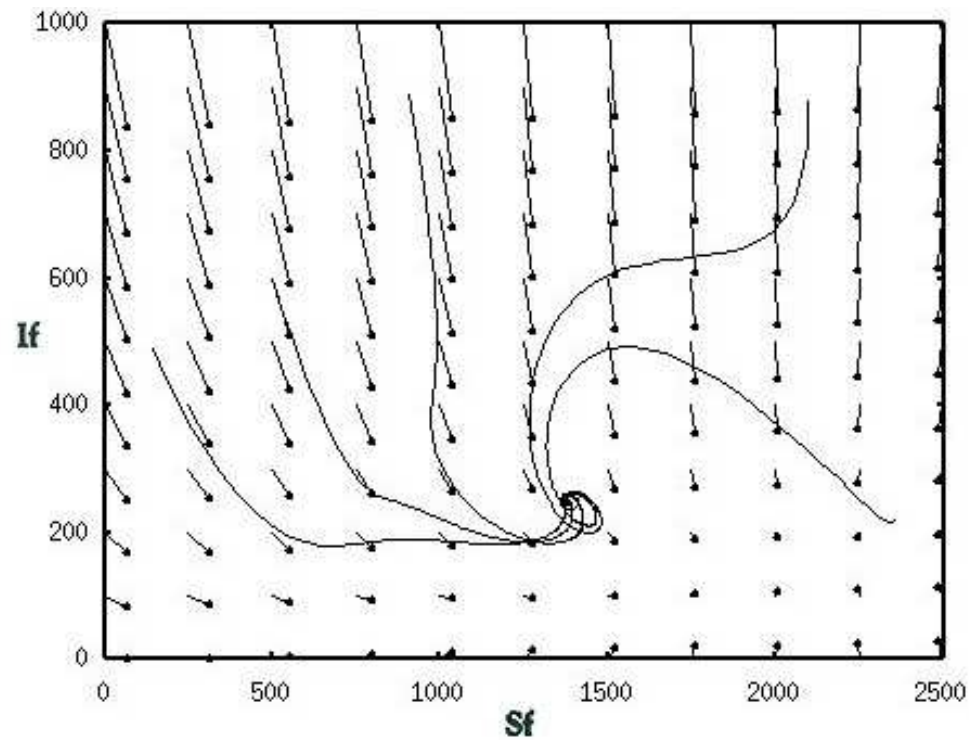

Fig. 5: The phase portrait of endemic equilibrium point $E_{1}$ in $S_{f}-I_{f}$ plane.

and treatment of HIV and other sexually transmitted infections for sex workers in developing and low income countries. As per these guidelines, the focus and emphasis is to be on working towards the decriminalization of sex workers. Furthermore, these guidelines have outlined a set of interventions that includes: 1) health services should be made available, accessible and acceptable to sex workers grounded on the principles of avoidance of stigma, non-discrimination; 2) to empower and make aware the sex workers and emphasize that correct and consistent use of condoms; 3) offering periodic screening for asymptomatic STIs to female sex workers; 4) voluntary HIV testing and counseling for couples and female sex workers. Certainly, all these can be helpful to reduce HIV transmission.

This paper has been motivated by the importance in understanding the role of female sex workers in transmission of the HIV an observation widely accepted in all the major reports of the WHO. In this paper, the disease-free equilibrium is globally stable for $R_{0}<1$ whereas the endemic equilibrium is globally stable for $R_{0}>1$. Our numerical simulations demonstrate that with 


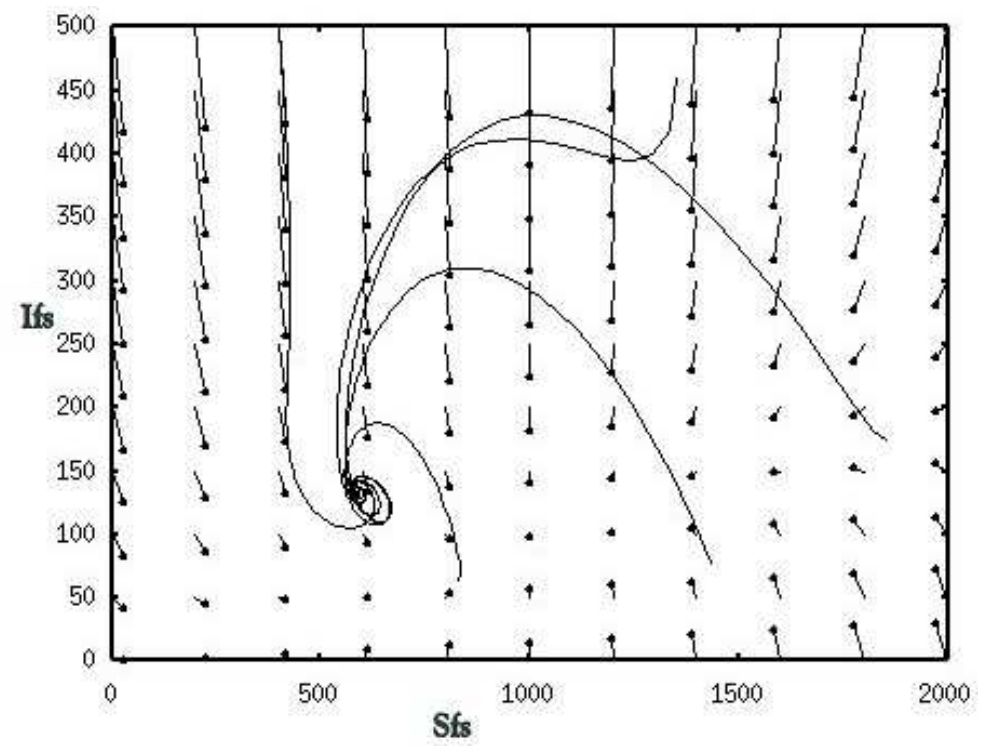

Fig. 6: The phase portrait of endemic equilibrium point $E_{1}$ in $S_{f s}-I_{f s}$ plane.

the increase in the rate of transmission due to female sex-workers, the total HIV infective population increases. Based upon the presented results, we conclude that to control the spread of HIV/AIDS epidemic, awareness among sex workers and their clients will play a very effective role. Hence, the need is to make preventive and informed services for the sex workers. More widespread prevention efforts at a larger level with proper coverage are required that include changing the social and legal context of the sex workers.

Additionally, from the available research reports we can observe that the stigma and discrimination attached to sex workers are barriers in effective and equitable health care and treatment. Furthermore, the criminalization of the sex workers restricts individuals from availing the health services which can improve their health and can increase their life span. In this regard, the available evidences suggest that the stigma reduction is critically important in efficient implementation of HIV prevention, care, and treatment policies [34].

We believe that the analysis reported in this paper can be strengthened by better understanding of limitations of the model and with appropriate real world data. The interventions program and policies need to be designed to guide and empower female sex workers to adopt healthier sex behavior and to reduce their risk of HIV infection. Our future work will go in this direction and currently, this is under investigation.

\section{Acknowledgement}

The authors thank the handling editor and anonymous referees for their valuable comments and suggestions which led to an improvement of our original manuscript.

\section{References}

[1] UNAIDS/WHO AIDS epidemic update, (2006). http://www.who.int/hiv/medicentre/2006EpiUpdateen.pdf.

[2] World Health Organization, (2006). TB/HIV, http://www.who.int/tb/hiv/en/.

[3] World Health Organization, (October 2013). Media centre. http://www.who.int/mediacentre/factsheets/fs360/en/.

[4] Boily MC, Baggaley RF, Wang L, Masse B, White RG, Hayes RJ, Alary M. Heterosexual risk of HIV-1 infection per sexual act: systematic review and meta-analysis of observational studies, The Lancet Infectious Diseases, 9, 118-129 (2009).

[5] Venkataramana, C. B. S., \& Sarada, P. V., Extent and speed of spread of HIV infection in India through the commercial sex networks: a perspective, Tropical Medicine and International Health, 6, 1040-1061 (2001).

[6] Gangakhedkar, R. R., Bentley, M. E., Divekar, A. D., Gadkari, D., Mehendale, S. M., Shepherd, M. E., Bollinger, R. C., \& Quinn, T.C., Spread of HIV infection in married monogamous women in India, JAMA., 278, 2090-2 (1997).

[7] George, S., Jacob, M., John, T. J., Jain, M. K., Nathan, N., Rao, P. S. S., Richard, J., \& Antonisamy, B., A casecontrol analysis of risk factors in HIV transmission in South India, Journal of Acquired Immune Deficiency Syndromes and Human Retrovirology, 14, 290-293 (1997).

[8] Panda, S., Chatterjee, A., Bhattacharya, S. K., Manna, B., Singh, P. N., Sarkar, S., Naik, T. N., Chakrabarti, S., \& Detels, R. Transmission of HIV from injecting drug users to their wives in India, International Journal of STD and AIDS, 11, 468-473 (2000). 


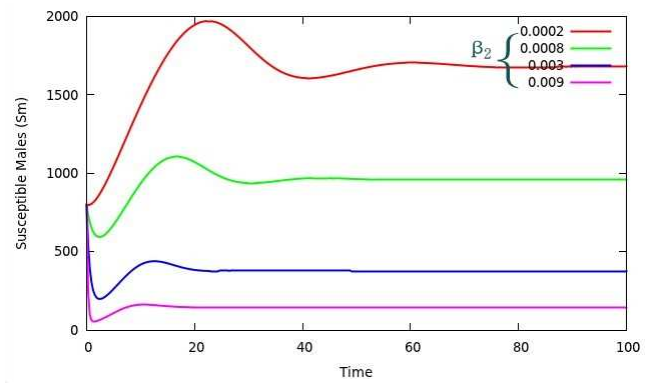

(a)

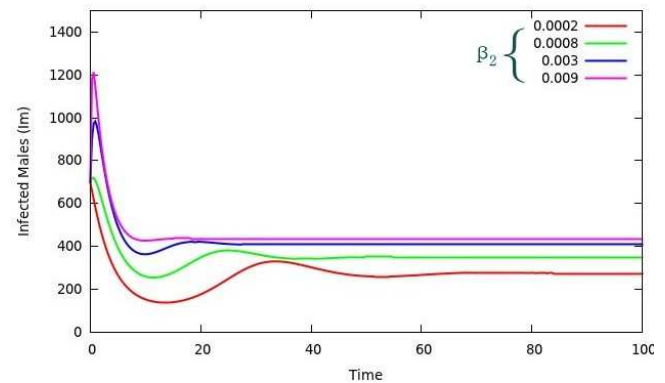

(b)

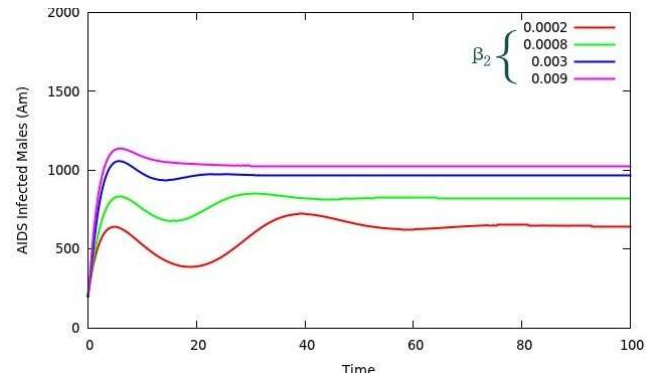

(c)

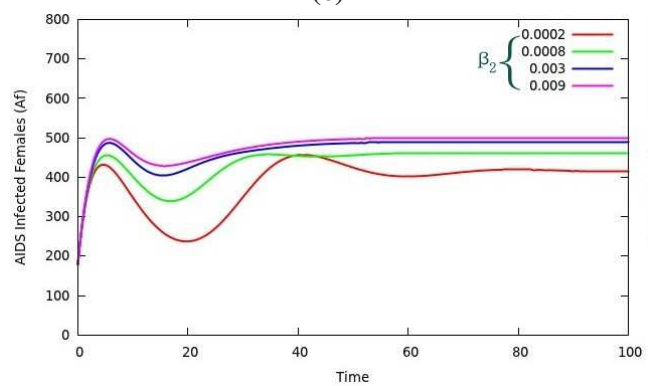

(d)

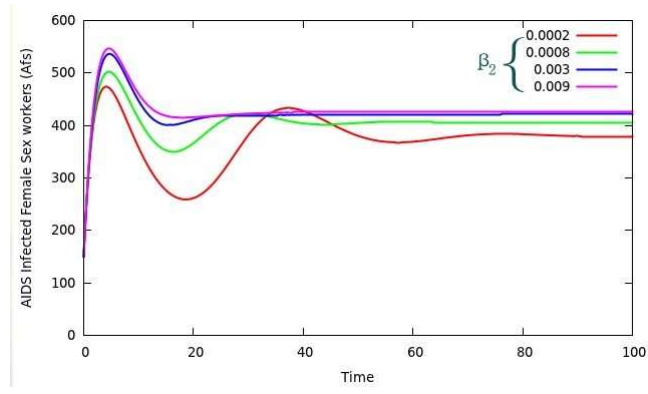

(e)

Fig. 7: Sensitivity of $S_{m}, I_{m}, A_{m}, A_{f}, A_{f s}$ for different values of $\beta_{2}$.
[9] Newmann, S., Sarin, P., Kumarasamy, N., Amalraj, E., Rogers, M., Madhivanan, P., Flanigan, T., Cu-Uvin, S., McGarvey, S., Mayer, K., \& Solomon, S. Marriage, monogamy and HIV: a profile of HIV-infected women in south India. International Journal of STD and AIDS, 11, 250253 (2000).

[10] Prevention and treatment of HIV and other sexually transmitted infections for sex workers in low- and middleincome countries, WHO Department of HIV/AIDS (2012). http://apps.who.int/iris/bitstream/10665/77745/1/9789241504744_eng.pdf.

[11] Stefan Baral, Prof Chris Beyrer, Kathryn Muessig, Tonia Poteat, Andrea L Wirtz, Michele R Decker, Susan G Sherman and Deanna Kerrigan, Burden of HIV among female sex workers in low-income and middle-income countries: a systematic review and meta-analysis. Lancet Infectious Diseases, 12, 538-549 (2012).

[12] Morison L, Weiss HA, Buv A, Caral M, Abega SC, Kaona F, Kanhonou L, Chege J and Hayes RJ, Commercial sex and the spread of HIV in four cities in sub-Saharan Africa. AIDS, 15, S61-S69 (2001).

[13] Michael L Rekart,. Sex-work harm reduction. Lancet, 366, 2123-2134 (2005).

[14] Bea Vuylsteke , Anjana Das , Gina Dallabetta and Marie Laga. Preventing HIV among sex workers. In: Mayer K, Pizer HF, eds. HIV prevention: a comprehensive approach. Burlington, MA, Academic Press, 376-406 (2012).

[15] Plummer FA et al., The importance of core groups in the epidemiology and control of HIV-1 infection. AIDS, 5, S169S176 (1991).

[16] Thomas JC, Tucker MJ., The development and use of the concept of a sexually transmitted disease core. Journal of Infectious Diseases, 174, S134-S143 (1996).

[17] G. P. Garnett,. An introduction to mathematical models in sexually transmitted disease epidemiology, Sex Transm Infect, 78, 7-12 (2002).

[18] Frank Ball, Peter Neal, A general model for stochastic SIR epidemics with two levels of mixing, Mathematical Biosciences, 180, 73-102 (2002).

[19] Frank Ball and Owen D. Lyne, Stochastic multi-type SIR epidemics among a population partitioned into households, Advances in Applied Probability, 33, 1-291 (2001).

[20] N. Kaur, M. Ghosh and S. S. Bhatia, Modeling the Spread of HIV in a Stage Structured Population: Effect of Awareness, International Journal of Biomathematics, 5, 250040 (2012).

[21] Tiina Roose, S. Jonathan Chapman, and Philip K. Maini, Mathematical Models of Avascular Tumor Growth, SIAM Rev., 49, 179-208 (2007).

[22] Gubler, D. J., Vector-borne diseases. Revue Scientifique et Technique - Office International des pizooties, 28, 583-588 (2009).

[23] Brauer, F., Z. Feng and C. Castillo-Chavez,. Discrete Epidemic Models, Mathematical Biosciences and Engineering, 7, 1-15 (2010).

[24] Grizzle, J.W., Abba, G., Plestan, F.; Asymptotically stable walking for biped robots: analysis via systems with impulse effects, Automatic Control, IEEE Transactions on, 46, 51-64 (2001).

[25] Roeger, Lih-Ing, W, Feng Z and C Castillo-Chavez, . The Impact of HIV infection on Tuberculosis, Mathematical Biosciences and Engineering, 6, 815-837 (2009). 
[26] L. Cai, X. Li, M. Ghosh,. Stability analysis of an HIV/AIDS epidemic model with treatment, J. Comp. and App. Math., 229, 313-323 (2008).

[27] Y. Hsieh and C. H. Chen,. Modelling the social dynamics of a sex industry: Its implications for spread of HIV I AIDS, Bulletin of Mathematical Biology, 66, 143-166 (2004).

[28] Rebecca F. Baggaley and Christophe Fraser, . Modelling sexual transmission of HIV: testing the assumptions, validating the predictions, Current Opinion in HIV and AIDS, 5, 269-276 (2010).

[29] A. Tripathi, R. Naresh and D. Sharma,. Modelling the effect of screening of unaware infectives on the spread of HIV infection, Appl. Math. Comp., 184, 1053-1068 (2007).

[30] Azim T, Chowdhury EI, Reza M, et al. Vulnerability to HIV infection among sex worker and non-sex worker female injecting drug users in Dhaka, Bangladesh: Evidence from the baseline survey of a cohort study. Harm Reduction Journal, 333 (2006).

[31] Njue C, Voeten HA, Remes P.. Disco funerals: A risk situation for HIV infection among youth in Kisumu, Kenya. AIDS., 23, 505-509 (2009).

[32] Tripathi BM, Sharma HK, Pelto PJ, Tripathi S., Ethnographic mapping of alcohol use and risk behaviors in Delhi. AIDS and Behavior., 14, S94-S103 (2010).

[33] Zapiola I, Salomone S, Alvarez A, et al., HIV-1, HIV2, HTLV-I/II and STD among female prostitutes in Buenos Aires, Argentina. Eur J Epidemiol., 12, 27-31(1996).

[34] Violence Against Women and HIV/AIDS: Critical Intersections http://www.who.int/gender/documents/sexworkers.pdf.

[35] Fenton K. A., Johnson A. M., McManus S, Erens B., Measuring sexual behaviour: methodological challenges in survey research, Sex Transm Infect, 77, 84-92 (2001).

[36] P. van den Driessche and J. Watmough, Reproduction numbers and sub-threshold endemic equilibria for compartmental models of disease transmission, Math. Biosci., 29-48 (2002).

[37] O. Diekmann, J.A.P. Heesterbeek, J.A.J. Metz, On the definition and the computation of the basic reproduction ratio $R_{0}$ in models for infectious diseases in heterogenous populations, J. Math. Biol., 28, 365-382 (1990).

[38] Castillo-Chavez C, Feng Z, Huang W,. On the computation of $R_{0}$ and its role on global stability. math.la.asu.edu/chavez/2002/JB276.pdf. (2002).

[39] J. P. LaSalle,. The stability of dynamical systems, Regional Conference Series in Applied Mathematics, SIAM, Philadelphia, PA, (1976).

[40] Bard Ermentrout, Simulating, Analyzing, and Animating Dynamical Systems: A Guide to XPPAUT for Researchers and Students, SIAM, Philadelphia, USA, (2002).

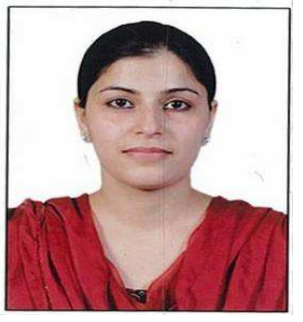

Navjot Kaur received the M.Sc (Master of Science) in Mathematics from Punjabi University, Patiala, India. Currently, she is pursuing her Ph.D (Doctor of Philosphy) in Mathematics, from the School of Mathematics and Computer Application, Thapar University, Patiala, India. Her current research interests are: Mathematical modeling, simulation and analysis of biological processes; nonlinear dynamics; and control theory.

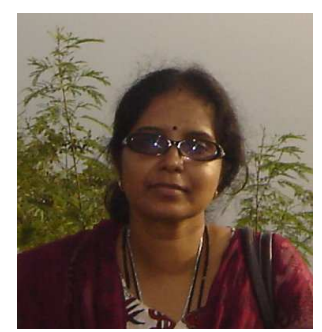

Mini Ghosh is an
Associate Professor at
the School of Advanced
Sciences, VIT University
Chennai Campus, India.
She received her Ph.D
in Mathematics from the IIT,
Kanpur, India in 2002. After
her doctoral degree she was a Post Doctoral Research Fellow at the Department of Mathematics, University of Trento, Italy from 2002-2004, and Institute of Information and Mathematical Sciences, Massey University, New Zealand from 2004-2006. Currently she is actively involved in many research problems, and specializes in the areas of Mathematical Modelling of epidemiological/ecological systems.



S. S. Bhatia is professor and former Head of School of Mathematics and Computer Applications, Thapar University, Patiala, India. He holds Ph.D in Mathematics from M. D. University, India. His areas of interest include functional analysis, real analysis, Fourier analysis, biomathematics, differential geometry and reliability analysis. Prof. Bhatia is author of numerous research articles, which have appeared in journals including Proceedings of the Indian Academy of Sciences - Mathematical Sciences, Journal of Mathematical Analysis and Applications, Applied Mathematics and Computation, Advanced Fuzzy Systems, International Journal of Biomathematics, Fixed Point Theory and Applications. 